\title{
Intensity self-pulsations in (GaAl)As injection lasers operating in an external cavity
}

\author{
Luis Figueroa \\ Hughes Research Laboratories, Malibu, California 90265 \\ Kam Lau and Amnon Yariv \\ California Institute of Technology, Pasadena, California 91125
}

(Received 12 October 1979; accepted for publication 4 December 1979)

\begin{abstract}
We present an experimental and theoretical study of a self-pulsing ( $\mathrm{GaAl}) \mathrm{As}$ injection laser operating in an external cavity. We have observed suppression of the self-pulsations when the external cavity is in the range $6<L<10 \mathrm{~cm}$. Suppression of self-pulsations can also be obtained by using a multimode graded index optical fiber as the external resonator. These results can be explained by a model which includes the effects of an external cavity and electron trapping. For long cavity lengths, the self-pulsation frequency locks to an external cavity harmonic with no significant quenching, as observed in some earlier experiments.
\end{abstract}

PACS numbers: 42.55.Px, 42.60.By

In recent months there has been renewed interest in studying intensity self-pulsatons in injection lasers. It has been reported that in a large percentage of aged lasers there is a tendency for self-sustaining oscillations to develop with a frequency ranging from $0.2-2 \mathrm{GHz}{ }^{1,2}$ The frequency of the oscillation decreases with increasing aging time and may lead to very undersirable consequences in communication or other signal processing systems. Several models ${ }^{1,3-9}$ have been proposed to explain the origins of the self-pulsations. Most of the present models assume that self-pulsations are produced by either a saturable absorber ${ }^{3,4,6,7}$ or a superlinear gain which increases with photon density.$^{1,8,9}$ The saturable absorption can arise from nonuniform current flow, ${ }^{3,4} \mathrm{ab}$ sorption centers distributed throughout the active region, ${ }^{6}$ or localized absorption centers near the laser facets. ${ }^{7}$ The superlinear gain can result from electron traps distributed throughout the active region ${ }^{1}$ or from a phenomenological origin. ${ }^{8,9}$ There exists little experimental data to verify any one model conclusively. The correlation between theory and experiment is further complicated by the different laser geometries and the mathematical similarities between the different models.

In a recent publication Chinone et al. ${ }^{10}$ reported on a method for the suppression of intensity pulsations by using a short external cavity $(0.3-2 \mathrm{~cm})$. Experimental results by Paoli et al." showed that the external cavity locks the selfpuslation frequency to a cavity harmonic, with no significant quenching effect. These results are not adequately explained by the conventional rate equations, which do not predict sustained pulsations. In this letter we report a study of a selfpulsing ( $\mathrm{GaAl}) \mathrm{As}$ injection laser operation in an external cavity. We use the conventional set of rate equations widely used to analyze relaxation oscillation in lasers. These are modified by the addition, in the manner of Copeland, ' of absorbing electron traps, and also by a term accounting for the feedback due to the external resonator. Using these equations, we show that the aforementioned observations are not independent, and fit well within the scope of a single model. ${ }^{12}$ Our analysis is confirmed by experimental results on a self-pulsing laser. Although we use Copeland's equations in our calculations, we believe that other well known models for self-pulsation, when modified to include the external cavity, would produce similar results. Second, we describe a novel method to suppress self-pulsations using an optical fiber resonator. The compactness of the laser-fiber system makes it a very attractive method for stabilizing self-pulsing lasers in practice.

The experiment consists of collimating the light from one facet of a Hitachi buried-heterostructure (BH) laser (HLP 2400), operating cw, using a $40 \times$ microscope objective. A portion of this light is returned to the laser by using a mirror mounted on a micrometer stage. The light from the other laser facet is focused onto a TI XL55 Avalanche diode (rise time $\sim 130 \mathrm{ps}$ ). The signal from the APD is amplified using $\mathrm{a} B$ and $\mathrm{H}$ amplifier with $3.1 \mathrm{GHz}$ bandwidth and 30$\mathrm{dB}$ gain. The signal is displayed on an HP 8565A Spectrum Analyzer. In the absence of an external cavity the $\mathrm{BH}$ laser self-pulses for currents slightly above threshold $(1>1.02$ $\left.I_{\mathrm{th}}\right)$. According to Copeland, self-pulsations are produced by electron traps distributed throughout the active region which can modulate the gain of the laser. The model is described by the following set of equations.

$$
\begin{aligned}
& \frac{d N_{\mathrm{e}}(t)}{d t}=\frac{J}{e d}-\frac{N_{\mathrm{c}}(t)}{\tau_{s}}-A N_{\mathrm{ph}}^{(t)}\left(N_{\mathrm{e}}-N_{\mathrm{o}}\right)+\frac{d T(t)}{d t}, \\
& \frac{d N_{\mathrm{ph}}(t)}{d t}=\frac{-N_{\mathrm{ph}}(t)}{\tau_{\mathrm{ph}}}+A N_{\mathrm{ph}}\left[N_{\mathrm{e}}^{(t)}-N_{0}\right]+\beta N_{t / \tau}^{(t)} \\
& -\sigma_{0} c_{0} N_{\mathrm{ph}}^{(t)}\left(T_{0}-T\right)+\frac{R^{\prime} N_{\mathrm{ph}}}{\tau_{\mathrm{ph}}^{\prime}}(t-\tau) \\
& \frac{d T(t)}{d t} \sigma_{0} c_{0} N_{\mathrm{ph}}^{(1)}\left(T_{0}-T\right)-\sigma_{\mathrm{e}} v N_{\mathrm{e}}^{(t)} T(t) .
\end{aligned}
$$

The first two equations with the exception of the last terms (those involving $T$ and $R^{\prime}$ ) are the conventional rate equations, with $N_{\mathrm{e}}\left(\mathrm{cm}^{-3}\right)$ being the electron density and $N_{\mathrm{ph}}\left(\mathrm{cm}^{-3}\right)$ the photon density. In these equations $J$ is the current desnity $\left(\mathrm{A} / \mathrm{cm}^{2}\right), e(C)$ is the electronic charge, and $d(\mathrm{~cm})$ is the active region thickness. The effect of transverse 


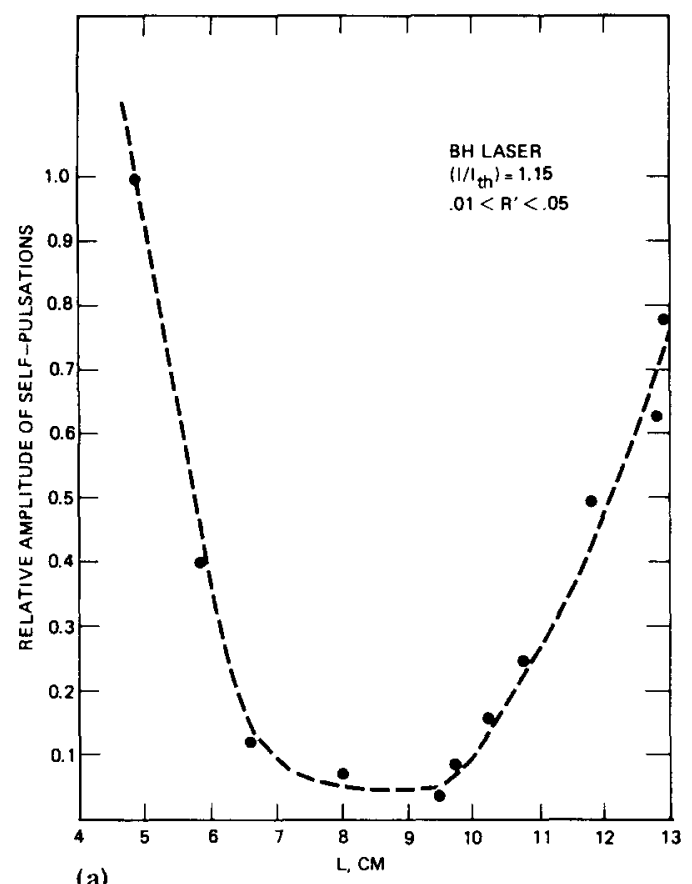

(a)

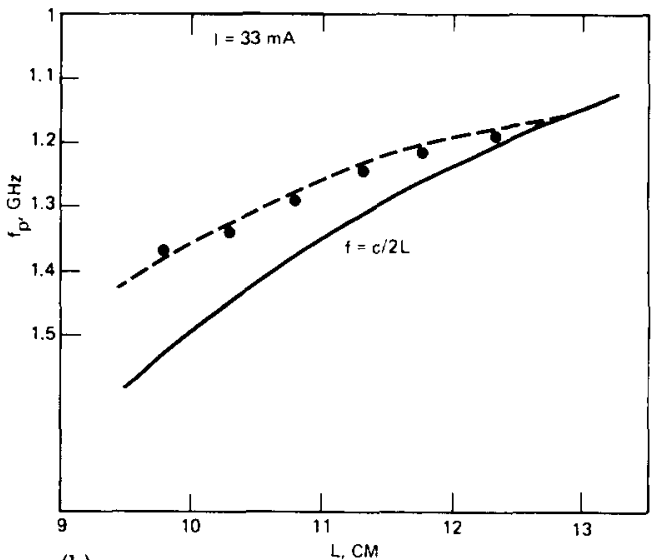

(b)
FIG. 1. (a) Amplitude of self-pulsations versus external cavity length, (b) frequency of self-pulsations versus cavity length. optical confinement and current spreading are neglected. The term $R^{\prime} N_{\mathrm{ph}}(t-\tau) / \tau_{\mathrm{ph}}^{\prime}$ represents the delayed feedback from the external mirror where $\tau$ represents the roundtrip time in the external caivty, $R^{\prime}$ is the fraction of light fed back into the laser, and $\tau_{\mathrm{ph}^{\prime}}$ only includes the mirror loss. The last equation represents the equation of motion for the traps

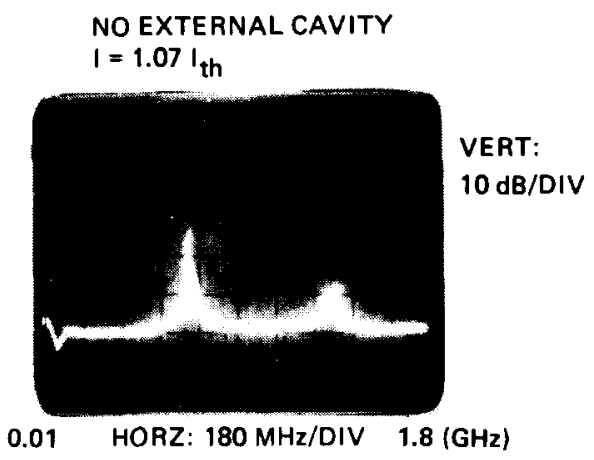

(a)

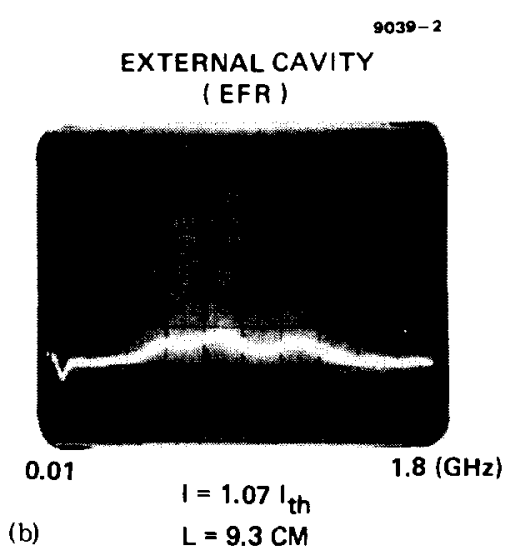

FIG. 2. (a) Photograph displaying self-pulsation with no external cavity, (b) same as (a), except a fiber resonator is aligned with the laser. where $c_{0}$ is the speed of light in the medium, $T$ is the density of empty traps, $T_{0}$ is the total number of traps, $\sigma_{e}$ is the electron capture cross section, $v$ is the thermal velocity, and $\sigma_{0}$ is the photon capture cross section by a trap occupied by an electron [whose density is $\left(T_{0}-T\right)$ ]. For the calculations we use typical values for the laser parameters: $A=1.5 \times 10^{-6}$ $\mathrm{cm}^{3} / \mathrm{c}, \tau_{\mathrm{s}}=3 \times 10^{-9} \mathrm{~s}, \tau_{\mathrm{ph}}=2.9 \times 10^{-12} \mathrm{~s}, d=1 \times 10^{-4} \mathrm{~cm}$, $N_{0}=5 \times 10^{17} \mathrm{~cm}^{-3}, \beta=10^{-4}, c_{0}=8 \times 10^{9} \mathrm{~cm} / \mathrm{sec}$, $\sigma_{e}=1.5 \times 10^{-17} \mathrm{~cm}^{2}, V=4.42 \times 10^{7} \mathrm{~cm} / \mathrm{sec}$, and $\sigma_{0}=3 \times 10^{-16} \mathrm{~cm}^{2}$. The calculations were performed by integrating Eqs. (1) to (3) using the Runge-Kutta fourth-order algorithm. The system is excited with a step change in current and run until steady-state oscillation or its absence is confirmed. A good fit of the experimental frequency and amplitude of the pulsations versus injection current can be obtained with an assumed trap density of $T_{0}=5.8 \times 10^{16} / \mathrm{cm}^{3}$.

Figure 1 shows an experimental plot of the amplitude and frequency of sefl-pulsation versus external cavity length $L$, for $I=1.15 I_{\text {th }}$. We note that there is a broad minimum in the amplitude of the self-pulsations for $6<L<10 \mathrm{~cm}$. Similar results are obtained for currents varying from $1.04 I_{\mathrm{th}}$ to $1.25 I_{\text {th }}$. An estimate of the coupling coefficient $R^{\prime}$ can be made from the measured reduction in threshold current when the external reflector is used and assuming that the mode gain $G$ can be approximated by $G=\beta\left(J-J_{0}\right)$. Our results indicate a coupling coefficient $R^{\prime}$ in the range $0.01-$ 0.05 . We find no significant variation in $R^{\prime}$ as the mirror is moved, indicating that the output laser beam is well collimated. In Fig. 2 we show the suppression of the self-pulsations using an external fiber resonator (EFR). The resonator consists of a piece of multimode graded index fiber with one end formed into a lens using the thermal melting technique ${ }^{13}$ while the other end is cleaved and $\mathrm{Au}$ is evaporated to form a reflector. The characteristics of injection lasers operating with an (EFR) have been discussed in more detail in Ref. 14. 


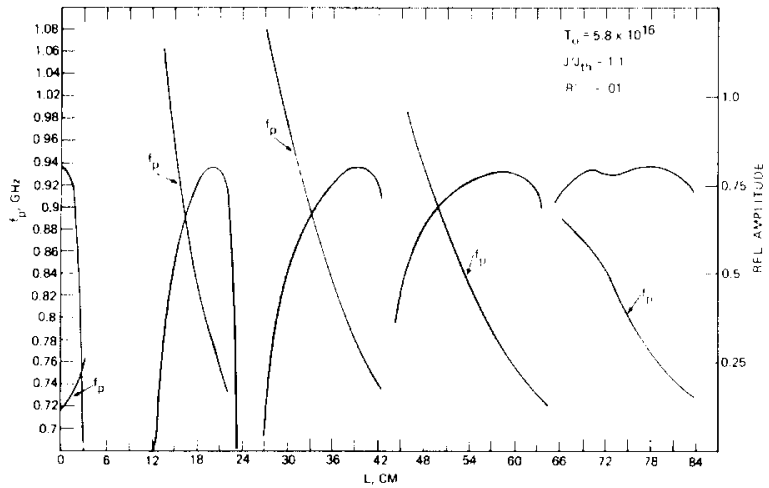

FIG. 3. Calculated plot of the self-pulsation amplitude and frequency versus cavity length.

When the fiber resonator is aligned with the injection laser and the length of the fiber is properly chosen, we are able to suppress self-pulsations with very little coupling into the (EFR) (i.e., the change in $I_{\mathrm{th}}$ is not measurable). The suppression of the self-pulsation is maintained for currents up to $1.1 I_{\mathrm{th}}$.

The calculated relative amplitude and frequency of the self-pulsations are plotted versus external cavity length in

Fig. 3. The value of $J$ is $1.1 J_{\text {th }}$ and $R^{\prime}=0.01$; however, the results do not change significantly for $0.01<R^{\prime}<0.2$. Several important observations can be pointed out. First, we find regions of $L$ where the self-pulsations are suppressed. The first band occurs for $3<L<12 \mathrm{~cm}$. This region roughly corresponds to our experimental results. In practice, the selfpulsation is not quenched completely in the suppressed region, leaving a resonance significantly broadened and greatly reduced in amplitude. In all the regions where the pulsations are not suppressed (except when $L<3 \mathrm{~cm}$ ), the frequency corresponds to $f_{p}=m c / 2 n L$ where $m$ is an integer, and $n$ is the refractive index. Starting with the first cavity harmonic, the pulsation locks to successive harmonics as $L$ is increased. Near the region where frequency jumping occurs, the oscillation is relatively unstable, without a well-defined period.

The results presented in Fig. 3 conform qualitatively to the experimental results of Paoli et al., " who used an external cavity approximately $75 \mathrm{~cm}$ in length. Our calculations show that the self-pulsation cannot be effectively suppressed for $L>50 \mathrm{~cm}$, and the pulsation frequency is locked to successive cavity harmonics as the cavity length is increased, as observed by Paoli et al. Chinone ${ }^{10}$ observed the quenching effect in a CSP laser, but for cavity lengths much shorter
$(0.3<L<2 \mathrm{~cm})$ than that predicted by our calculations. We believe the results obtained by Chinone could be explained by using a shorter photon lifetime in the calculations.

The mechanism involved in the suppression of the selfpulsations is the locking action produced by the external cavity feedback. By introducing external feedback, the injection laser is forced to pulsate at a frequency $f_{p}$ equal to $m c / 2 n L$. As $L$ is decreased, the laser is forced to pulsate at higher frequencies. If $\tau$, (i.e., $1 / f_{p}$ ) is shorter than the time required to replenish the electrons consumed in generatiing an optical pulse, then the pulsation cannot be sustained. As expected, higher pump currents can sustain pulsations at a higher frequency, and thus a shorter cavity is necessary for quenching. For very short cavity lengths $(L<3 \mathrm{~cm})$ the pulse width is comparable to the transit time of the light in the external cavity and no frequency locking occurs. Thus the pulsation is primarily determined by the parameters of the injection laser. On the other hand, if $L$ is too long, the self-pulsation frequency will lock to a higher cavity harmonic, with a frequency above the natural pulsation frequency and below the quenching frequency.

In conclusion we have demonstrated that self-pulsations in a cw injection laser can be suppressed by an external cavity. We have demonstrated that a useful external cavity for this purpose can be made using an optical fiber. Lastly, we performed numerical calculations using the electron-trap model of Copeland to confirm our experimental results and predict that suppression of self-pulsations in lasers operating in an external cavity occurs over a small range in external cavity lengths.

The authors are grateful to E. Lin for some help in the computer programming, T.R. Ranganath and H.W. Yen for discussions.

'J.A. Copeland, Electron. Lett. 14, 809 (1978)

${ }^{2} \mathrm{G}$. Arnold and K. Peterman, Opt. Quantum Electron. 10, 311 (1978).

${ }^{3}$ N.G. Basov, IEEE J. Quantum Elect. QE-4, 855 (1968).

${ }^{4}$ T.P. Lee and H.R. Roldan, IEEE J. Quantum Electron. QE-6, 339 (1970).

${ }^{5}$ T.L. Paoli and J.E. Ripper, Appl. Phys. Lett. 15, 105 (1969).

${ }^{6}$ D. Kato, Appl. Phys. Lett. 9, 588 (1977).

${ }^{7}$ R.W. Dixon and W.B. Joyce, IEEE J. Quantum Electron. QE-15, 470 (1979).

${ }^{8}$ J.P. Van der Ziel, J.L. Merz, and T.L. Paoli, J. Appl. Phys. 7, 4620 (1979).

${ }^{9}$ B.W. Hakki, J. Appl. Phys. 9, 5630 (1979).

${ }^{10}$ N. Chinone, K. Aiki, and R. Ito. Appl. Phys. Lett. 33, 990 (1978)

"'T.L. Paoli and J.E. Ripper, Appl. Phys. Lett. 6, 335 (1970).

${ }^{12}$ The authors have recently become aware of the work by $H$. Kuwahara [Appl. Phys. 20, 67 (1979)], who discusses the effect of an external cavity on the amplitude of self-pulsations.

${ }^{13}$ D. Kato, J. Appl. Phys. 44, 2756 (1973).

${ }^{14}$ L. Figueroa, C. Slayman, and H.W. Yen, Hughes Research Lab, quarterly report, prepared for Naval Research Laboratory under contract N0017378-C-0192, April 1979. 